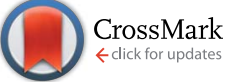

Cite this: RSC Adv., 2015, 5, 12365

Received 5th November 2014

Accepted 15th January 2015

DOI: 10.1039/c4ra13901a

www.rsc.org/advances

\section{The effects of solvent composition on the affinity of a peptide towards hair keratin: experimental and molecular dynamics data $\uparrow$}

\author{
Egipto Antunes, Célia F. Cruz, Nuno G. Azoia and Artur Cavaco-Paulo*
}

\begin{abstract}
The study of the interaction between hair filaments and formulations or peptides is of utmost importance in fields like cosmetic research. Keratin intermediate filament structure is not fully described, limiting the molecular dynamics (MD) studies in this field despite its high potential to improve the area. We developed a computational model of a truncated protofibril, simulated its behavior in alcoholic based formulations and with one peptide. The simulations showed a strong interaction between the benzyl alcohol molecules of the formulations and the model, leading to the disorganization of the keratin chains, which regress with the removal of the alcohol molecules. This behavior can explain the increase of peptide uptake in hair shafts evidenced in fluorescence microscopy pictures. The model developed is valid to computationally reproduce the interaction between hair and alcoholic formulations and provide a robust base for new MD studies about hair properties. It is shown that the MD simulations can improve hair cosmetic research, improving the uptake of a compound of interest.
\end{abstract}

\section{Introduction}

Hair is a proteinaceous biomaterial with a complex structure, serving to protect the body against environmental factors such as cold or heat, the sunlight and wetness.

The hair fiber is usually divided into three zones: the cuticle (outermost zone, Fig. 1G), the cortex (surrounded by the cuticle), and in the center zone of thicker hairs, the medulla (Fig. 1F). The cuticle corresponds to a stratified layer of thin and flat overlapping cells, like fish scales, and acts as a chemical and physical barrier, protecting the inner zones. The medulla is constituted by hollow cells with amorphous material and air spaces. ${ }^{1}$

The cortex has long, cylindrical assembled cortical cells surrounded by the cell membrane complex (Fig. 1A). Macrofibrils (Fig. 1B) and intermacrofibrilar material composes the cortical cells. The macrofibrils can be further divided into microfibrils (Fig. 1D) and a surrounding matrix (Fig. 1C) composed of associated proteins, cytoplasmatic and nuclear remains of keratinocytes. The microfibrils are also known as intermediate filaments, and are composed of 4 protofibrils (Fig. 1E), which have 2 protofilaments, each one with two

CEB - Centre of Biological Engineering, University of Minho, 4710-057 Braga, Portugal.E-mail: artur@deb.uminho.pt

$\dagger$ Electronic supplementary information (ESI) available: Details about the keratin protofibril model built, pictures of the time evolution of the systems in the interactions simulations, and information about the benzyl alcohol parametrization are described in this file. See DOI: 10.1039/c4ra13901a dimers of $\alpha$-helix coiled-coil keratins. Fig. 1 illustrates the hierarchical structure of the hair fiber.

In the intermediate filaments assembly, the $\alpha$-helix coiledcoil dimer of keratin is made with one keratin of type I (acidic keratins) and one of type II (basic or neutral keratins), forming a heterodimer, since this assembly is much more stable than a homodimer. $^{2}$ Each $\alpha$-keratin has four coiled-coil domains (denominated as 1A, 1B, 2A and 2B), three linker domains (L1, L12, and L2), and the head and tail terminal domains. This structure is shared with all intermediate filaments such as vimentin, desmin and lamins, and is represented in Fig. 2.

The assembly of the keratin dimer is made by pairing each domain of one $\alpha$-keratin with the same domain of the adjacent

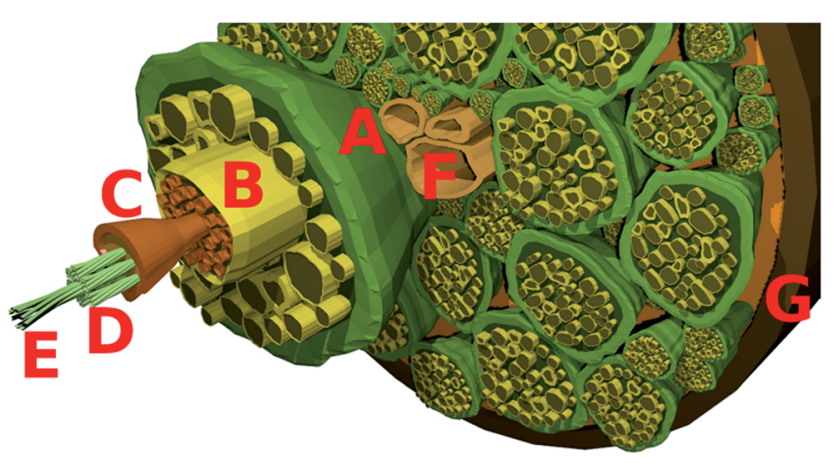

Fig. 1 Schematic 3D representation of one cut hair shaft. (A): Cell membrane complex; (B): macrofibril; (C): matrix; (D): microfibrils or intermediate filaments; (E): protofibril; $(F)$ : hollow medulla cells; $(G)$ : hair cuticle. The representation is not at scale. 


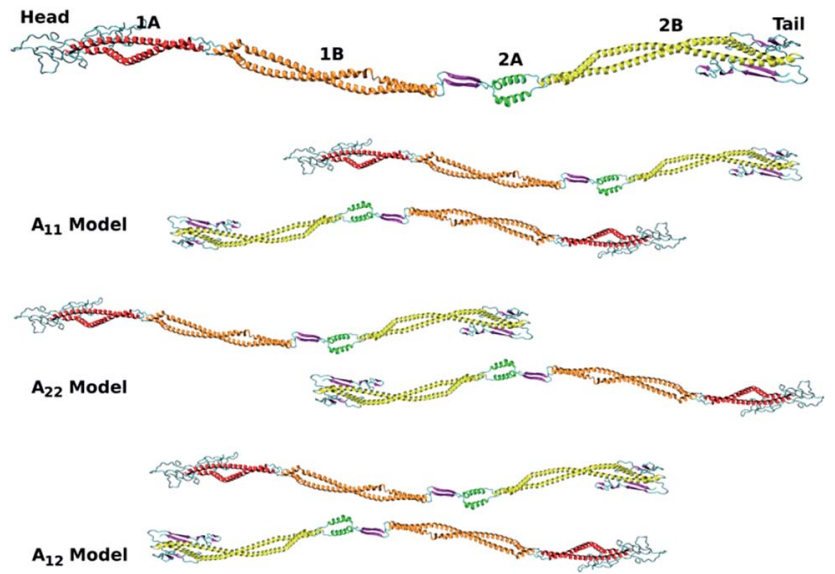

Fig. 2 Top: Representation of one vimentin intermediate filament dimer, with its domains discriminated. Bottom: Representation of three models of intermediary filaments dimers pairing. The pictures were rendered using the structure obtained by Zhao Qin and colleagues. ${ }^{3}$

$\alpha$-keratin (see Fig. 2 Top), while the assembly of one protofilament (two dimers) is anti-parallel. It is not completely clear how the protofilament assembly is made, and there are three main modes of pairing described, namely the $A_{11}, A_{12}$ and the $A_{22}$ (see Fig. 2). ${ }^{4}$ The $A_{11}$ mode is defined by the antiparallel overlap of $1 B$ segments, the $\mathrm{A}_{22}$ mode by the antiparallel overlap of $2 \mathrm{~B}$ segments and the $\mathrm{A}_{12}$ mode by the antiparallel overlap of intermediate filaments dimers. The higher assemblies in octamers (protofibrils), micro and macrofibrils of the IFs, are not fully characterized, at the molecular level, mainly in the hair IFs. The few literature about this thematic, incised more in the vimentin IFs due the existence of crystallographic models for these filaments, or in the skin keratins due to the skin diseases that affect many humans, ${ }^{5-8}$ however these filaments are different from hair IFs.

There are 54 elements of the human keratin family (skin and hair keratins), 28 of type I and 26 of type II. It was found that keratin 31, 33a, 34, 38b, 81, 85 and 86 are the most abundant keratins in the hair ${ }^{9}$ and, for example, keratin 31 is mostly expressed in lower and mid cortex, while keratins 34 and 86 are mostly expressed in mid and upper cortex. ${ }^{\mathbf{1 0 - 1 2}}$ It seems that the pairing of the protofilament is not predefined in terms of the keratins elements, allowing competition between the keratins and different pairs can be found. ${ }^{\mathbf{1 2}}$

Molecular dynamics (MD) has been applied with success in the study of complex biomaterials, providing unique and detailed molecular information inaccessible by others techniques. The systems studied by MD are increasing in number, diversity and complexity, from single proteins in water, ${ }^{13}$ to cell membranes ${ }^{14,15}$ or even stratum corneum. ${ }^{16-18}$

Despite all potential of MD simulations, this technique has only been applied in the study of hair in a few works. ${ }^{19-22}$ The MD has a great potential to boost some aspects in the hair products development research, speeding up the process while saving resources and money. Previously our group studied the affinity between fragments of pulmonary associated surfactant protein D (SPD) and $\alpha$-keratin, in formulations containing benzyl alcohol, propylene glycol and ethanol. ${ }^{19}$ SPD is a surfactant protein synthesized in the lung and due to the ability of these kind of peptides to interact with hair lipids they have been study as helper agents in the recovery of damaged hair. ${ }^{19,23,24}$ Here we are extending the scope of this previous work by building a bigger computational model of a hair and changing formulations composition. The new model describe a truncated protofibril ( 8 keratins), assembling the $2 \mathrm{~B}$ domains of keratin 34 and keratin 86, as a simplified model of one hair shaft. This model was subject to several MD simulations with different formulations. The SPD fragment chosen was the one with less affinity to keratin in the previous work, to facilitate the evaluation of differences in peptide affinity due to the formulations. The simulations results were compared with experimental tests performed with real hair. We intent to show that the affinity of peptides can be increased by formulations composition and that this property, together with the stronger interactions of the formulations with the hair, can be assessed by MD simulations.

\section{Materials and methods}

All simulations were performed with the GROMACS 4.0.7 (ref. 25) software package, using the MARTINI ${ }^{26}$ force field. The MARTINI force-field, being a coarse-grained force-field, has a possible drawback when dealing with proteins, namely the rigidity of its secondary structure, thus not allowing the modeling of secondary structure changes. However, there are many examples of studies of proteinaceous systems with this force field with great success, since the secondary structure rigidity only limits studies, focusing on properties or phenomena, strongly dependent on the protein structure..$^{27-29}$

In all simulations the procedure was the same, namely we begun with the energy minimization of the systems for 500 steps with steepest descent method. Then the systems were subject to a short simulation ( $3 \mathrm{~ns}$ ), in the canonical ensemble or NVT (number of molecules, volume and temperature constants), to relax the structures and generate velocities according to a temperature of $300 \mathrm{~K}$. The production simulations were performed with the isothermal-isobaric ensemble or NPT (number of molecules, pressure and temperature constants), with simulation times ranging from 65 to $510 \mathrm{~ns}$. Berendsen coupling was used to maintain the temperature at $300 \mathrm{~K}$ with a relaxation time of $0.7 \mathrm{ps}$, and also a Berendsen coupling to maintain the pressure at $1 \mathrm{~atm}$, with a time constant of 3 ps and a compressibility of $3 \times 10^{-5}$ bar $^{-1} .^{30}$ The simulation box was dodecahedric, the integration time step of $30 \mathrm{fs}$ and the Lincs algorithm was used as bond constrainer. ${ }^{31}$ To the non-bonded interactions, cut offs and shifts at $12 \AA$ were applied, with a decrease of the van der Waals forces between 9 and $12 \AA$, and a decrease of electrostatic forces throughout the whole interaction distance.

\section{Keratin protofibril model building}

The majority of the hair weight corresponds to the keratin fiber, but a complete macrofibril, or even the smaller microfibril 
reach sizes far beyond the usual system sizes simulated in MD. However the hair has a hierarchical structure, in which smaller units will assemble into bigger structures and these assemble again into even bigger structures, giving the macroscopic properties of the material. ${ }^{21,32}$ Considering this we built a computational model of a truncated protofibril, assembling 8 truncated $\alpha$-keratins. The truncated zone corresponds to $\alpha$ keratins $2 \mathrm{~B}$ domain from the $\mathrm{L} 2$ link to $\mathrm{C}$ tail, and the assembly of the 4 dimers is based in the $\mathrm{A}_{22}$ model, with dimer to dimer anti-parallel pairing. Pymol software ${ }^{33}$ was used to create the $3 \mathrm{D}$ all-atom structure of one keratin 34 (type I) and of one keratin 86 (type II), using its peptide sequence from UniProt database. The choice of the keratin chains was based in the fact that both belong to the group of the most expressed keratins in hair, and both are present on the hair cortex, increasing the probability of this pair be representative of hair cortex content. The A22 model was chosen because there is not a consensus about the model that better describe the two dimer pairing, and this model allows the end to end pairing of the different chains turning the full structure more compact. These structures were converted to coarse-grained models, and were duplicated and joined together to build the dimer, the tetramer and finally the octamer. The resulting model of one truncated protofibril of hair keratin was chosen taking into account the physical assembling properties presented in MD simulations, comparing with the existing studies about hair keratins and intermediate filaments assembling:, ${ }^{\mathbf{3 , 6 , 3 4}}$ A more detailed description of the full process is presented in ESI. $\dagger$

\section{Interactions simulations}

The hair model thus obtained was then used in the simulations of the interaction of several formulations with the hair fiber. The formulations tested were based on the one used in the previous work of our group, ${ }^{19}$ and are composed by ethanol, benzyl alcohol, propylene glycol and water. The original formulation (OF) had a composition (v/v) of 10, 0.5, 1.5 and 88, respectively. The computational formulations used in this work do not include explicit PGO due to limitations with its parametrization to the MARTINI force field. In this force field four water molecules are modeled by one polar bead, P4 type, and the parametrization of the propylene glycol molecule would lead to one polar bead similar to the four water model. The ethanol parametrization is available, together with other solvents in the MARTINI website (http://md.chem.rug.nl/ cgmartini/), and this molecule is modeled by a P1 bead. For benzyl alcohol there is no parametrization available, so we modeled the molecule, based on the available benzene parametrization, as three SC5 beads, corresponding to the benzene like ring, and one P1 bead corresponding to the $\mathrm{OH}$ alcohol group. More details about this alcohol parametrization are described in ESI. $\dagger$

The concentration of benzyl alcohol ranged between 0.5 and $3 \%(\mathrm{v} / \mathrm{v})$, since the benzyl alcohol solubility limit in water is 3.5 $\mathrm{g} / 100 \mathrm{~mL}$ at $20{ }^{\circ} \mathrm{C}$ and $4.29 \mathrm{~g} \mathrm{~mL}^{-1}$ at $25{ }^{\circ} \mathrm{C}$, and solutions with $4 \%$ of this alcohol present some turbidity (see Fig. 3 and 4 in $\mathrm{ESI} \dagger$ material).
The behavior of the hair model in the formulations was simulated for $510 \mathrm{~ns}$, with the same protocol described above. After the simulations the systems evolution and the final structure of the protofibrils were evaluated either visually or statistically, measuring properties such as the radial distribution function (RDF), the root mean square deviation (RMSD), the radius of gyration (RG) and the solvent accessible surface area (SAS).

Besides the experimental tests, described in the next section, we also assess the effect of BE3, and B3 formulations in the interaction of one SPD fragment (QAAFSQ) with the hair through MD. This peptide corresponds to a small fragment of a glycoprotein synthesized by alveolar type II cells and by nonciliated bronchiolar epithelial cells ${ }^{35}$ and was chosen because the previous work had demonstrated low affinity with keratin dimer. Thus it is expected that the increase of affinity of this peptide with hair will be easier to demonstrate. We create one system for each formulation, with 4 replicas each, containing the hair model and 6 peptides. The behavior of the systems was simulated for $510 \mathrm{~ns}$, and in the end the formulations were removed from the simulation box, water was added and the behavior of the aqueous systems was simulated for another $510 \mathrm{~ns}$.

We also performed some preparatory simulations of the protofibril and the BE3 formulation using the gromos 43A1-S3 force field ${ }^{36}$ with the same methodology of the coarse grained simulations This united atom force field provides atomic detail to the system however turns the system huge and more computing demanding. The results of these simulations were similar to the coarse grained simulations (see $\mathrm{ESI} \uparrow$ material, Fig. 5), but we only obtained computing performances of $0.4 \mathrm{~ns} /$ $24 \mathrm{~h}$ against $50 \mathrm{~ns} / 24 \mathrm{~h}$ in the coarse grained simulations. Once the lack of atomic detail seems do not affect the properties studied in the coarse grained simulations (the results of united atoms and coarse grained simulations are similar), and these simulations are much less computing demanding (allowing simulate longer time scales), we advance in the work with only the coarse grained simulations.

\section{Experimental tests}

The interaction simulations were compared with experimental tests in human hair. Virgin Asian hair tresses were provided by International Hair Importers \& Products (New York). Before use, the hair fibers were washed with running water and commercial shampoo. The hair tresses undergone eight cycles of bleaching because in our research group we intend to develop products to help in damaged hair recovering, and the bleaching although very common in hair styling is a very aggressive treatment to the hair. In each cycle, the hair was incubated with $10 \% \mathrm{H}_{2} \mathrm{O}_{2}(\mathrm{v} / \mathrm{v})$ in $\mathrm{Na}_{2} \mathrm{CO}_{3} / \mathrm{NaHCO}_{3} \mathrm{pH} 9.0$, for 1 hour at $50{ }^{\circ} \mathrm{C}$. Between cycles, the hair was washed with running water and commercial shampoo.

Afterwards, the different formulations, whose compositions are described in Table 1 were tested in the bleached hair fibers.

In order to ease the analysis of the formulation effect, we use the engineered SPD fragment covalently linked, in the 
Table 1 Description of the different aqueous formulations content used in this work. The percentage is relative to the solvent volume. BE: formulations containing benzyl alcohol and ethanol; B: formulations containing benzyl alcohol

\begin{tabular}{llll}
\hline & & \multicolumn{2}{c}{ Ethanol } \\
Label & Benzyl alcohol & Vol (\%) & Propylene glycol \\
\hline OF & 0.5 & 10 & 1.5 \\
BE0.5 & 0.5 & 10 & 0 \\
BE1 & 1 & 10 & 0 \\
BE1.5 & 1.5 & 10 & 0 \\
BE2 & 2 & 10 & 0 \\
BE2.5 & 2.5 & 10 & 0 \\
BE3 & 3 & 10 & 0 \\
B1.5 & 1.5 & 0 & 0 \\
B2 & 2 & 0 & 0 \\
B2.5 & 2.5 & 0 & 0 \\
B3 & 3 & 0 & 0
\end{tabular}

N-terminus, to the fluorescent dye 5(6)-carboxytetramethylrhodamine, succinimidyl ester (or 5-TAMRA). This peptide was synthesized by JPT Peptide Technologies GmbH (Berlin, Germany). After treatment cross sections of the hair fibers samples were analyzed by fluorescence microscopy. The hair fibers were initially embedded in an epoxy resin and $15 \mathrm{~mm}$ transversal cuts were obtained using a microtome (Leitz). The samples were analyzed using an inverted fluorescence microscope Leica DMIL at a magnification $20 \times$. The most representative images were chosen.

\section{Results and discussion}

The non-existence of an entire crystallographic model of the keratin intermediate filaments is likely the major responsible for the low number of MD studies focusing the hair. To overcome this limitation, models of keratin and of the larger structures resulting of its assembling, such as dimers or protofilaments, can be designed and developed with more or less complexity and detail, and this approach was done on the few studies available. ${ }^{19-22}$

We joined 4 dimers of truncated keratin 34-keratin 86 chains (from the L2 link to C tail) and simulate its assembling in water, to build a truncated protofibril model (see $\mathrm{ESI}_{\dagger} \dagger$ file for more information). The assembled keratinaceous octamer in the end of these simulations presented structural properties similar to the literature description of the intermediate filaments. Since the precise determination of protofibril atomic structure is out of the scope of this work, we move ahead for the interaction simulations.

The time evolution of the system BE4 is depicted in the Fig. 3. It is clear the destabilization of the fiber, which is promoted by the formulation, since the initial protofibril structure is stable in water. It is also clear the tendency of the benzyl alcohol molecules to be located near the hair model, interacting with its keratins. Both effects are also evident in all other systems, with and without ethanol, and the destabilization seems to increase with the benzyl alcohol concentration (see Fig. 1 and 2 in ESI $\dagger$ ).

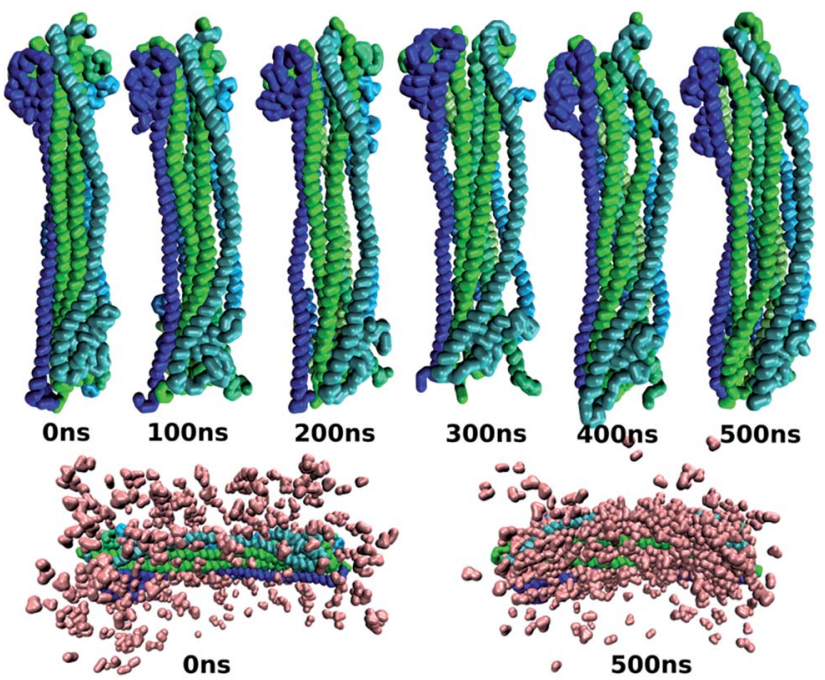

Fig. 3 Top: Time evolution of the hair model (truncated protofibril) incubated in the formulation BE3. The solvent molecules were omitted for clarity. Bottom: Initial and final states of the same system showing the tendency of benzyl alcohol molecules (pink) to interact with the chains of keratin.

The RDF analysis confirms the benzyl alcohol tendency to be located near the hair model. This analysis defines the ratio between the local and average concentration of the target molecules, at a given distance. The value 1 indicates a random distribution of the molecules, and higher values indicate a tendency of the molecules to be located at such distance. The RDF analysis of systems BE2 and B2 is presented in the Fig. 4. All the other systems presented similar RDF distribution pattern.

It is clear that the RDFs values for benzyl alcohol molecules are very high at small distances, with some initial peaks corresponding to solvation layers, decreasing for bigger distances. For higher distances the RDF values are lower than 1, confirming that the benzyl alcohol molecules tend to be located near to the hair, as it was shown in Fig. 3. The ethanol and water behavior are remarkably distinct to benzyl alcohol, while similar to each other; the RDFs values in both cases are lower than 1 , increasing towards this value as the distance increase. The absence of ethanol does not change the RDF behavior of both water and benzyl alcohol.

The degree of destabilization observed in the simulations appears to increase with the concentration of the benzyl alcohol in the systems. However it is hard to visually evaluate the differences between systems with lower variation of benzyl alcohol concentration, problem that is overcome by the structural analysis of the hair model in the system.

The RMSD indicates the spatial deviation of the hair structure from its starting conformation. The average values from the final $110 \mathrm{~ns}$ of simulation in the different systems are presented in the Table 2 .

The systems presented an overall increase of RMSD values with the increase of benzyl alcohol content, and there are no significant differences between the systems with or without ethanol. The RMSD values show a substantial spatial deviation 


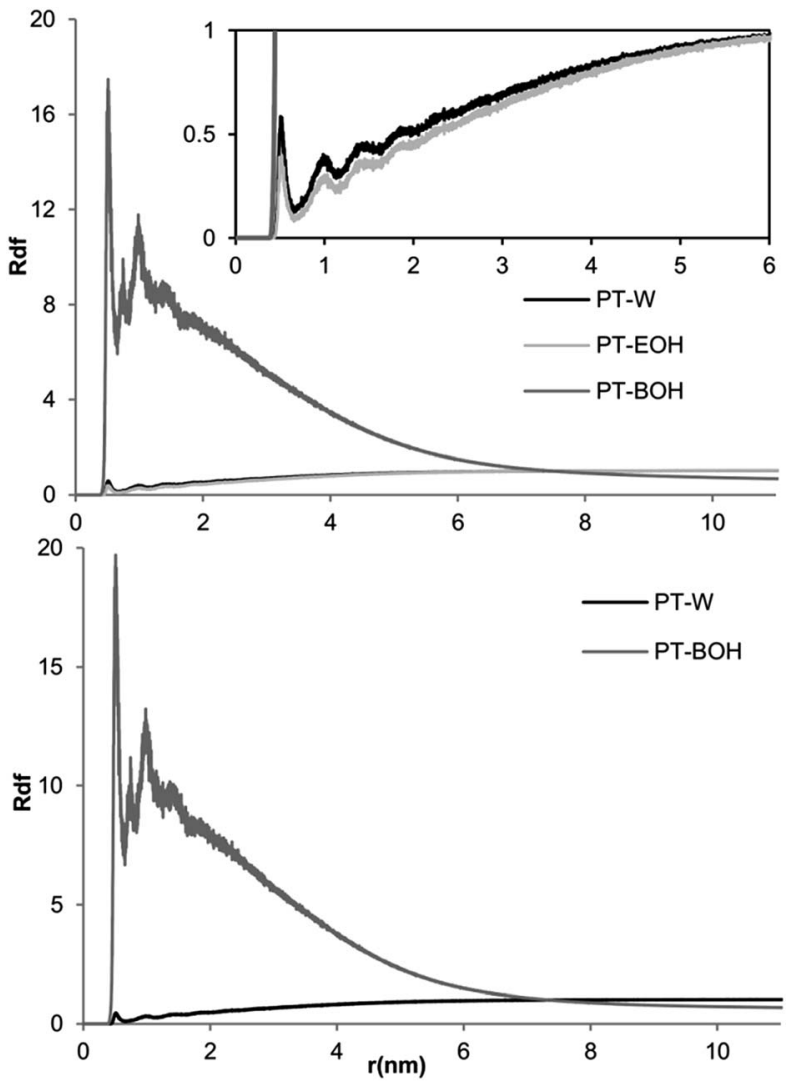

Fig. 4 Graphical representation of the radial distribution function (RDF) values of the systems with $2 \%$ of benzyl alcohol, with (Top) and without (Bottom) ethanol molecules. PT-W, PT-EOH, PT-BOH:RDF values between the protofibril and water; ethanol and benzyl alcohol, respectively.

of the protofibril initial structure, which seems to be linked to the benzyl alcohol concentration. However the evaluation of RMSD values cannot be directly linked to the destabilization observed in the simulations because movements like torsions, rotations, and stretching also contribute to the RMSD values but do not lead to the lateral dissociation observed in the simulations.

To better understand the effect of the formulations in the stability of the hair model, the RG and SAS values were evaluated. These values are presented in the Fig. 5. The SAS analysis provides information about the area of the hair model that is accessible to the solvent, being related with the hair model destabilization because the lateral dissociation of the keratins increases the area of the proteinaceous material accessible to

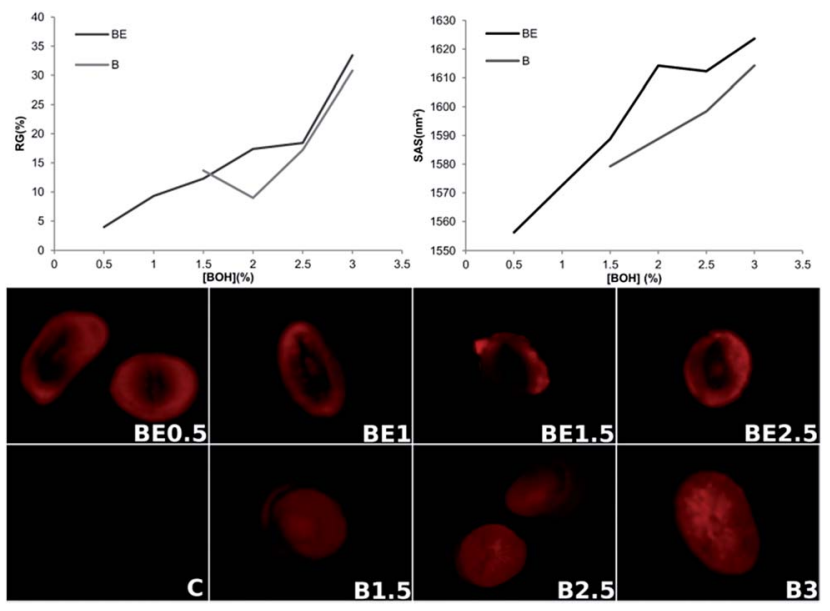

Fig. 5 Top: Graphical representation of the Radius of Gyration (RG) and Solvent Accessible Surface area (SAS). Bottom: Snapshots of transversal cuts of bleached hair shafts, treated with the different formulations and with the QAAFSQ-TAMRA peptide (C: control).

the solvent. On the other hand, in MD studies, the RG measures the compaction of the structure of proteins. We measured the RG relative to the transversal axis of the cylindrical protofibril, and the values were normalized, which allows the evaluation of the lateral disassembling of the keratins chains since it follows this axis.

The RG and SAS values showed a global tendency to increase with the increase of benzyl alcohol concentration. As in the RMSD analysis, are bigger the differences between the RG and SAS values in the lower and higher benzyl alcohol concentration than between the RG and SAS values in formulations with or without ethanol.

The fluorescence microscopy pictures in the Fig. 5 clearly showed an increase of fluorescence in the hair. The pictures showed one interesting feature - the hair treated with formulations containing ethanol presented much lower fluorescence in the inner zones of hair cortex. At this point it is clear that the formulations composition will affect the stability of the keratins assembly in the hair shaft, and that this destabilization is able to ease the penetration of the peptide into the hair. In order to evaluate, at a molecular level, the interaction of the peptide with the hair model some simulations were performed. Some snapshots of the evolution of one system composed by the hair model and 6 SPD fragments, during $1020 \mathrm{~ns}$, are presented in Fig. 6. In the first half of the simulation the proteinaceous material was dissolved in the BE3 formulation and the second half only in water.

Table 2 Root Mean Square Deviation (RMSD) average values of the last 110 ns of each simulation and the respectively standard deviation $\operatorname{RMSD}(\mathrm{nm})$

\begin{tabular}{|c|c|c|c|c|c|}
\hline BE0.5 & BE1 & BE1.5 & BE2 & BE2.5 & BE3 \\
\hline $0.83 \pm 0.04$ & $1.03 \pm 0.03$ & $1.10 \pm 0.04$ & $1.25 \pm 0.03$ & $1.26 \pm 0.02$ & $1.19 \pm 0.04$ \\
\hline & & B1.5 & B2 & B2. 5 & B3 \\
\hline
\end{tabular}


Once again the formulations promote the destabilization of the keratin assembly, allowing the penetration of the peptide into the interior of the fiber. Nonetheless the interaction of the peptide with the keratins is not very strong while the formulation is present, but when the formulations are removed, what is equivalent to washing the hair after the incubation with the formulations, the peptide stays attached to the fiber. What seems to be clear is that the formulations do not increase the affinity of the peptide towards the hair. Instead, the formulations are able to facilitate the penetration of the peptide into the fiber, increasing the amount of peptide that penetrates the fiber and interacts with the keratins. This behavior is in agreement with the fluorescence pictures, showing how the peptide can be absorbed by the hair. The same simulations were made for the formulations B3 (results not showed), and the behavior was similar, the lateral expansion of the keratins in hair model was bigger when the formulations do not contain ethanol, and this can be the cause of the difference in fluorescence patterns showed in the microscopy studies. Bigger expansions can lead

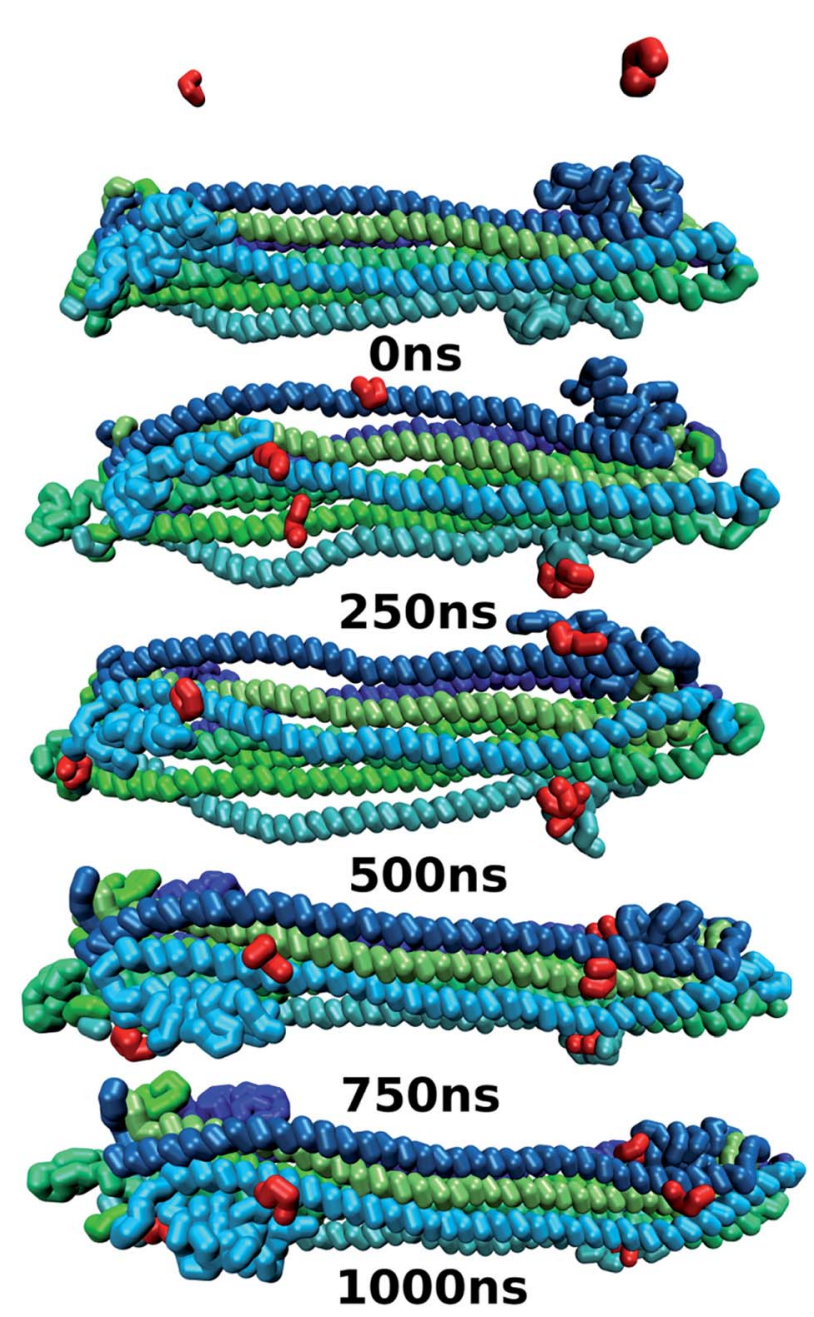

Fig. 6 Snapshots of the simulation of the hair model and 6 peptides of QAAFSQ (in red) in the BE3 formulation until 510 ns. The BE3 formulation was removed at this point and the system was filled with water, extending the simulation for another 510 ns. to more peptide being trapped in the hair fibers, while allowing the peptide to penetrate deeper into the hair shaft.

The small solubility range of benzyl alcohol in aqueous solutions limited the number of different formulations is this work. This is a limitation of the experimental procedure, were we are using aqueous solutions to mimic cosmetic formulations. We also made simulations with higher concentration of benzyl alcohol (see ESI Fig. 1 and $2 \dagger$ ), that were excluded from the work because the impossibility to compare with fluoresce tests, due to the solubility problems. However, in the cosmetic field the formulations used in commercial products are not solutions, but are instead gels, lotions, shampoos, among others, where the concentration of benzyl alcohol could be higher than its solubility in water. With the simulations it is possible to overcome solubility problems like this one, turning the screening of new formulations easier than in other models, like the ones using aqueous solutions.

\section{Conclusions}

The computational model built in this work proved to be a good approach to provide information about the way how formulations can change the affinity of peptides, or other compounds, towards hair fibers. The simulations showed a strong interaction between molecules of benzyl alcohol and the keratins, leading to a destabilization of the hair model structure which increases with the benzyl alcohol concentration. The fluorescence microscopy experiment showed that the absorption in the hair fiber of the SPD fragment is improved with the increase of benzyl alcohol in the formulations, and this can be explained by the mechanisms observed in the simulations. The theoretical and experimental data are in good agreement, showing that the built model is valid to describe the interactions between formulations and hair.

With the MD simulations we were able to select formulations with better performance in the enhancement of peptide absorption by the hair. But the hair model built allows the study of many other features and phenomena, as long as its inherent peculiarities are taken into account. This can be of great interest to researchers in fields of applied research, such as cosmetic applications, to fundamental research.

\section{Acknowledgements}

The authors acknowledge the access to the Minho University GRIUM cluster.

\section{Notes and references}

1 R. Dawber, Clin. Dermatol., 1996, 14, 105-112.

2 M. Hatzfeld, J. Cell Biol., 1990, 110, 1199-1210.

3 Z. Qin, L. Kreplak and M. J. Buehler, PLoS One, 2009, 4, e7294.

4 S. V. Strelkov, H. Herrmann and U. Aebi, BioEssays, 2003, 25, 243-251.

5 H. Herrmann, M. Häner, M. Brettel, N. O. Ku and U. Aebi, J. Mol. Biol., 1999, 286, 1403-1420. 
6 C.-H. Lee, M.-S. Kim, B. M. Chung, D. J. Leahy and P. a. Coulombe, Nat. Struct. Mol. Biol., 2012, 19, 707-715.

7 L. Norlén, S. Masich, K. N. Goldie and A. Hoenger, Exp. Cell Res., 2007, 313, 2217-2227.

8 U. Aebi, W. E. Fowler, P. Rew and T. T. Sun, J. Cell Biol., 1983, 97, 1131-1143.

9 N. R. Barthélemy, A. Bednarczyk, C. Schaeffer-Reiss, D. Jullien, A. Van Dorsselaer and N. Cavusoglu, Anal. Biochem., 2012, 421, 43-55.

10 J. Schweizer, L. Langbein, M. a. Rogers and H. Winter, Exp. Cell Res., 2007, 313, 2010-2020.

11 L. Langbein, J. Biol. Chem., 1999, 274, 19874-19884.

12 L. Langbein, M. a. Rogers, H. Winter, S. Praetzel and J. Schweizer, J. Biol. Chem., 2001, 276, 35123-35132.

13 A. C. Lorenzo and E. R. Caffarena, J. Biomech., 2005, 38, 1527-1533.

14 A. P. Demchenko and S. O. Yesylevskyy, Chem. Phys. Lipids, 2009, 160, 63-84.

15 E. Antunes, N. G. Azoia, T. Matamá, A. C. Gomes and A. Cavaco-Paulo, Colloids Surf., B, 2013, 106, 240-247.

16 C. Das, M. G. Noro and P. D. Olmsted, Biophys. J., 2009, 97, 1941-1951.

17 M. I. Hoopes, M. G. Noro, M. L. Longo and R. Faller, J. Phys. Chem. B, 2011, 115, 3164-3171.

18 M. Martins, N. G. Azoia, A. Ribeiro, U. Shimanovich, C. Silva and A. Cavaco-Paulo, Colloids Surf., B, 2013, 108, 271-278.

19 N. G. Azoia, M. M. Fernandes, N. M. Micaêlo, C. M. Soares and A. Cavaco-Paulo, Proteins: Struct., Funct., Bioinf., 2012, 80, 1409-1417.

20 C. Danciulescu, B. Nick and F.-J. Wortmann, Biomacromolecules, 2004, 5, 2165-2175.

21 C.-C. Chou and M. J. Buehler, Biomacromolecules, 2012, 13, 3522-3532.
22 D. W. Cheong, F. C. H. Lim and L. Zhang, Langmuir, 2012, 28, 13008-13017.

23 M. Fernandes and A. Cavaco-Paulo, Biocatal. Biotransform., 2012, 30, 10-19.

24 M. M. Fernandes and A. Cavaco-Paulo, Biocatal. Biotransform., 2012, 30, 111-124.

25 B. Hess, C. Kutzner, D. van der Spoel and E. Lindahl, J. Chem. Theory Comput., 2008, 4, 435-447.

26 S. J. Marrink, H. J. Risselada, S. Yefimov, D. P. Tieleman and A. H. de Vries, J. Phys. Chem. B, 2007, 111, 7812-7824.

27 B. A. Hall, J. P. Armitage and M. S. P. Sansom, PLoS Comput. Biol., 2012, 8, e1002685.

28 H. J. Risselada, G. Marelli, M. Fuhrmans, Y. G. Smirnova, H. Grubmüller, S. J. Marrink and M. Müller, PLoS One, 2012, 7, e38302.

29 H. J. Risselada, C. Kutzner and H. Grubmüller, ChemBioChem, 2011, 12, 1049-1055.

30 H. J. C. Berendsen, J. P. M. Postma, W. F. van Gunsteren, A. DiNola and J. R. Haak, J. Chem. Phys., 1984, 81, 3684.

31 B. Hess, H. Bekker, H. J. C. Berendsen and J. G. E. M. Fraaije, J. Comput. Chem., 1997, 18, 1463-1472.

32 C. Popescu and H. Höcker, Chem. Soc. Rev., 2007, 36, 12821291.

33 The PyMOL Molecular Graphics System, Version 1.7.1.3, Schrödinger, LLC.

34 S. V. Strelkov, H. Herrmann, N. Geisler, T. Wedig, R. Zimbelmann, U. Aebi and P. Burkhard, EMBO J., 2002, 21, 1255-1266.

35 A. Persson, D. Chang, K. Rust, M. Moxley, W. Longmore and E. Crouch, Biochemistry, 1989, 28, 6361-6367.

36 S.-W. Chiu, S. A. Pandit, H. L. Scott and E. Jakobsson, J. Phys. Chem. B, 2009, 113, 2748-2763. 